International Mathematical Forum, Vol. 8, 2013, no. 3, 113 - 124

\title{
Synchronization of a Restricted Three Body Problem under the Effect of Radiation Pressure
}

\author{
Ayub Khan \\ Department of Mathematics \\ Zakir Husain College \\ University of Delhi \\ Delhi-110007, India \\ Priyamvada Tripathi \\ Department of Mathematics \\ University of Delhi \\ Delhi-110007, India \\ dupriyam@gmail.com
}

\begin{abstract}
In this paper we have investigated the synchronization behavior of a restricted three body problem under the effect of radiation pressure evolving from different initial conditions using active control technique based on the Lyapunov-stability theory and Routh-Hurwitz criteria. The designed controller with our own choice of coefficient matrix of error dynamics are found to be effective in the stabilization of error states at the origin, thereby synchronization between the state variables of two dynamical systems under the consideration. Numerical simulations are presented to illustrate the effectiveness of the proposed control techniques using mathematica.
\end{abstract}

Keywords: Restricted three body problem under the effect of radiation pressure, Synchronization, Lyapunov stability theory and Routh- Hurwitz criteria.

\section{Introduction}

Synchronization is the dynamical process by which two or more oscillators adjust their rhythms due to a weak interaction [1]. This problem has received the great attention in the literature due to its importance in engineering and physical sciences, as well as in the challenging biological and social entities 
$[1,2,3]$. Chaotic synchronization did not attract much attention until Pecora and Carroll [4] introduced a method to synchronize two identical chaotic systems with different initial conditions in 1990 and they demonstrated that chaotic synchronization could be achieved by driving or replacing one of the variables of a chaotic system with a variable of another similar chaotic device. From then on, enormous studies have been done by researchers on the synchronization of dynamical systems. In the last two decades considerable research has been done in non-linear dynamical systems and their various properties. One of the most important properties is synchronization. Here, synchronization in restricted three body problem under the effect of radiation pressure has been focused. Restricted three body problem attracted a great attention of researchers for a long time. Apparently, Euler was the first who introduced the notion of the restricted three body problem in [5], devoted to the motion of the Earth and the Moon relative to the Sun. It was possible to neglect the masses of the Earth and the Moon relative to the Sun mass, and also the smallness of the Moon and Earth masses ratio was taken into account. Neglect of the mass of one body compared with the masses of two other bodies simplifies essentially the solution of the problem. In addition, Bruns and Poincare [6] proved that the general solution of the three-body problem cannot be represented by algebraic or one-valued transcendental functions of coordinates and velocities of the bodies. In the research on the three-body problem, Poincare became the first person to discover a chaotic deterministic system [7] and underlined a possibility of chaotic behaviour in three body problem. A well known modification of the RTBP is that of Sitnikov [8] and stabilization of chaotic behavior in restricted three body problem on the example of a perturbed Sitnikov case has been studied in 2006 [9]. In general, this problem is related to stabilization and control of unstable and chaotic behavior of dynamical systems by external forces.

Synchronization techniques have been improved in recent years and many different methods are applied theoretically as well as experimentally to synchronize the chaotic systems, including adaptive control [10, 11, 12], backstepping design $[13,14,15]$, active control $[16,17,18]$ nonlinear control $[19,20,21$, $22]$ and observer based control method [23, 24]. Using these methods, numerous synchronization problem of well-known chaotic systems such as Lorenz, Chen, Lü and Rössler system have been worked on by many researchers. Recently, Ge et al. [25, 26, 27, 28, 29, 30] also studied chaotic synchronization of many practical physical systems and obtained interesting results. Among these methods chaos synchronization using active control has recently been widely accepted because it can be used to synchronize identical as well as non-identical systems; a feature that gives it an advantage over other methods.

In this paper, active control techniques base on the Lyapunov stability theory and Routh-Hurwitz criteria have been used to study the synchronization 
behavior of RTBP under the effect of radiation pressure. The system under consideration is chaotic for some values of parameter involved in the system. Here two systems (master and slave) are synchronized and start with different initial conditions. This problem may be treated as the design of control laws for full chaotic slave system using the known information of the master system so as to ensure that the controlled receiver synchronizes with master system. Hence the slave chaotic system completely traces the dynamics of the master system in the course of time.

The aim of this study to investigate the synchronization behavior of RTBP under the effect of radiation pressure.

\section{Equations of motion}

Equations of motion of restricted three body problem under the effect of radiation pressure are [31]:

$$
\begin{aligned}
& \ddot{x}-2 \dot{y}=x-(1+\mu) g_{1} \frac{(x+\mu)}{r_{1}^{3}}-\mu g_{2} \frac{(x-1+\mu)}{r_{2}^{3}}, \\
& \ddot{y}+2 \dot{x}=y-(1+\mu) g_{1} \frac{y}{r_{1}^{3}}-\mu g_{2} \frac{y}{r_{2}^{3}} \\
& \ddot{z}=-(1+\mu) g_{1} \frac{z}{r_{1}^{3}}-\mu g_{2} \frac{z}{r_{2}^{3}}
\end{aligned}
$$

with

$$
r_{1}^{2}=(x+\mu)^{2}+y^{2}+z^{2} \quad \text { and } \quad r_{2}^{2}=(x-1+\mu)^{2}+y^{2}+z^{2} .
$$

where $r_{1}$ and $r_{2}$ are the distances between third body and primaries. $g_{i} ; i=1,2$ are generic notation for handling the general problem, introduced by Elipe (1992), and

$$
g_{i}=\frac{\partial G_{i}}{\partial\left(1 / r_{i}\right)}
$$

where $G_{i}$ functions depending on the distances $r_{i}, i=1,2$, and $\mu=f m_{2}$, with $f$ the gravitational constant and $m_{2}$ is the mass of one of the primaries. 


\section{Synchronization via Active Control}

The system defined by (1) can be written as a system of six first order differential equations. The six variables are introduced as below:

$$
\left.\begin{array}{l}
x_{1}=x \\
x_{2}=\dot{x} \\
x_{3}=y \\
x_{4}=\dot{y} \\
x_{5}=z \\
x_{6}=\dot{z}
\end{array}\right\}
$$

From (1) and (2),

$$
\begin{aligned}
& \dot{x}_{1}=x_{2}, \\
& \dot{x}_{2}=x_{1}+2 x_{4}-(1+\mu) g_{1} \frac{\left(x_{1}+\mu\right)}{r_{1}^{3}}-\mu g_{2} \frac{\left(x_{1}-1+\mu\right)}{r_{2}^{3}}, \\
& \dot{x}_{3}=x_{4}, \\
& \dot{x}_{4}=x_{3}-2 x_{2}-(1+\mu) g_{1} \frac{x_{3}}{r_{1}^{3}}-\mu g_{2} \frac{x_{3}}{r_{2}^{3}}, \\
& \dot{x}_{5}=x_{6}, \\
& \dot{x}_{6}=-(1+\mu) g_{1} \frac{x_{5}}{r_{1}^{3}}-\mu g_{2} \frac{x_{5}}{r_{2}^{3}}
\end{aligned}
$$

with

$$
r_{1}^{2}=\left(x_{1}+\mu\right)^{2}+x_{3}^{2}+x_{5}^{2} \quad \text { and } \quad r_{2}^{2}=\left(x_{1}-1+\mu\right)^{2}+x_{3}^{2}+x_{5}^{2}
$$

corresponding to master system (3), the identical slave system is defined as:

$$
\begin{aligned}
& \dot{y}_{1}=y_{2}+u_{1}(t) \\
& \dot{y}_{2}=y_{1}+2 y_{4}-(1+\mu) g_{1} \frac{\left(y_{1}+\mu\right)}{r_{1}^{3}}-\mu g_{2} \frac{\left(y_{1}-1+\mu\right)}{r_{2}^{3}}+u_{2}(t) \\
& \dot{y}_{3}=y_{4}+u_{3}(t) \\
& \dot{y}_{4}=y_{3}-2 y_{2}-(1+\mu) g_{1} \frac{y_{3}}{r_{1}^{3}}-\mu g_{2} \frac{y_{3}}{r_{2}^{3}}+u_{4}(t) \\
& \dot{y}_{5}=y_{6}+u_{5}(t) \\
& \dot{y}_{6}=-(1+\mu) g_{1} \frac{y_{5}}{r_{1}^{3}}-\mu g_{2} \frac{y_{5}}{r_{2}^{3}}+u_{6}(t)
\end{aligned}
$$

with

$$
r_{1}^{2}=\left(y_{1}+\mu\right)^{2}+y_{3}^{2}+y_{5}^{2} \quad \text { and } \quad r_{2}^{2}=\left(y_{1}-1+\mu\right)^{2}+y_{3}^{2}+y_{5}^{2} .
$$

where $u_{i}(t) ; i=1,2,3,4,5,6$ are control functions to be determined. 
Now defining error functions such that in synchronization state $\lim _{t \rightarrow \infty} e_{i}(t) \rightarrow 0, i=1,2,3,4,5,6$.

$$
\begin{aligned}
& e_{1}=y_{1}-x_{1} \\
& e_{2}=y_{2}-x_{2} \\
& e_{3}=y_{3}-x_{3} \\
& e_{4}=y_{4}-x_{4} \\
& e_{5}=y_{5}-x_{5} \\
& e_{6}=y_{6}-x_{6}
\end{aligned}
$$

and error dynamics are expressed as:

$$
\left.\begin{array}{l}
\dot{e}_{1}=\dot{y}_{1}-\dot{x}_{1} \\
\dot{e}_{2}=\dot{y}_{2}-\dot{x}_{2} \\
\dot{e}_{3}=\dot{y}_{3}-\dot{x}_{3} \\
\dot{e}_{4}=\dot{y}_{4}-\dot{x}_{4} \\
\dot{e}_{5}=\dot{y}_{5}-\dot{x}_{5} \\
\dot{e}_{6}=\dot{y}_{6}-\dot{x}_{6}
\end{array}\right\}
$$

From equations (3), (4) and (5), we get

$$
\begin{aligned}
& \dot{e}_{1}(t)=e_{2}(t)+u_{1}(t) \\
& \dot{e}_{2}(t)=2 e_{4}(t)+e_{1}(t)-e_{1}(t)\left\{\frac{(1+\mu) g_{1}}{r_{1}^{3}}+\frac{\mu g_{2}}{r_{2}^{3}}\right\}+u_{2}(t) \\
& \dot{e}_{3}(t)=e_{4}(t)+u_{3}(t) \\
& \dot{e}_{4}(t)=-2 e_{2}(t)+e_{3}(t)-e_{3}(t)\left\{\frac{(1+\mu) g_{1}}{r_{1}^{3}}+\frac{\mu g_{2}}{r_{2}^{3}}\right\}+u_{4}(t) \\
& \dot{e}_{5}(t)=e_{6}(t)+u_{5}(t) \\
& \dot{e}_{6}(t)=-e_{5}(t)\left\{\frac{(1+\mu) g_{1}}{r_{1}^{3}}+\frac{\mu g_{2}}{r_{2}^{3}}\right\}+u_{6}(t) .
\end{aligned}
$$

The error dynamical system (6) to be controlled must be a linear system with control inputs. Therefore we redefine the control functions such as to eliminate non-linear terms in $e_{1}(t), e_{2}(t), e_{3}(t), e_{4}(t), e_{5}(t)$ and $e_{6}(t)$ of equation 
(6) as follows:

$$
\left.\begin{array}{l}
u_{1}(t)=v_{1}(t) \\
u_{2}(t)=e_{1}(t)\left\{\frac{(1+\mu) g_{1}}{r_{1}^{3}}+\frac{\mu g_{2}}{r_{2}^{3}}\right\}+v_{2}(t) \\
u_{3}(t)=v_{3}(t) \\
u_{4}(t)=e_{3}(t)\left\{\frac{(1+\mu) g_{1}}{r_{1}^{3}}+\frac{\mu g_{2}}{r_{2}^{3}}\right\}+v_{4}(t) \\
u_{5}(t)=v_{5}(t) \\
u_{6}(t)=e_{5}(t)\left\{\frac{(1+\mu) g_{1}}{r_{1}^{3}}+\frac{\mu g_{2}}{r_{2}^{3}}\right\}+v_{6}(t) .
\end{array}\right\}
$$

using (6) and (7), we have

$$
\begin{aligned}
& \dot{e}_{1}(t)=e_{2}(t)+v_{1}(t) \\
& \dot{e}_{2}(t)=2 e_{4}(t)+e_{1}(t)+v_{2}(t) \\
& \dot{e}_{3}(t)=e_{4}(t)+v_{3}(t) \\
& \dot{e}_{4}(t)=-2 e_{2}(t)+e_{3}(t)+v_{4}(t) \\
& \dot{e}_{5}(t)=e_{6}(t)+v_{5}(t) \\
& \dot{e}_{6}(t)=v_{6}(t)
\end{aligned}
$$

Equation (8) is the error dynamics, which can be interpreted as a control problem where the system to be controlled is a linear system with control inputs $v_{1}(t), v_{2}(t), v_{3}(t), v_{4}(t), v_{5}(t)$ and $v_{6}(t)$. We choose $v_{1}(t), v_{2}(t), v_{3}(t)$, $v_{4}(t), v_{5}(t)$ and $v_{6}(t)$ as follows:

$$
\left(\begin{array}{l}
v_{1}(t) \\
v_{2}(t) \\
v_{3}(t) \\
v_{4}(t) \\
v_{5}(t) \\
v_{6}(t)
\end{array}\right)=A\left(\begin{array}{l}
e_{1}(t) \\
e_{2}(t) \\
e_{3}(t) \\
e_{4}(t) \\
e_{5}(t) \\
e_{6}(t)
\end{array}\right)
$$

where $A$ is $6 \times 6$ constant feedback matrix to be determined. The error dynamical system (8) can be written as:

$$
\left(\begin{array}{l}
\dot{e}_{1}(t) \\
\dot{e}_{2}(t) \\
\dot{e}_{3}(t) \\
\dot{e}_{4}(t) \\
\dot{e}_{5}(t) \\
\dot{e}_{6}(t)
\end{array}\right)=B\left(\begin{array}{l}
e_{1}(t) \\
e_{2}(t) \\
e_{3}(t) \\
e_{4}(t) \\
e_{5}(t) \\
e_{6}(t)
\end{array}\right)
$$


where $B$ is $6 \times 6$ co-efficient matrix. According to the Lyapunov stability theory and Routh-Hurwitz criteria, eigen-values of the co-efficient matrix of error system must be real or complex with negative real parts. We can choose elements of matrix arbitrarily, there are several ways to choose in order to satisfy Lyapunov and Routh-Hurwitz criteria. Consequently, for

$$
\begin{aligned}
A & =\left(\begin{array}{cccccc}
-1 & -1 & 0 & 0 & 0 & 0 \\
-1 & -1 & 0 & -2 & 0 & 0 \\
0 & 0 & -1 & -1 & 0 & 0 \\
0 & 2 & -1 & -1 & 0 & 0 \\
0 & 0 & 0 & 0 & -1 & -1 \\
0 & 0 & 0 & 0 & 0 & -1
\end{array}\right) \\
B & =\left(\begin{array}{cccccc}
-1 & 0 & 0 & 0 & 0 & 0 \\
0 & -1 & 0 & 0 & 0 & 0 \\
0 & 0 & -1 & 0 & 0 & 0 \\
0 & 0 & 0 & -1 & 0 & 0 \\
0 & 0 & 0 & 0 & -1 & 0 \\
0 & 0 & 0 & 0 & 0 & -1
\end{array}\right)
\end{aligned}
$$

becomes a matrix with eigen values having negative real parts. Equation (9) with equation (10) reduces to,

$$
\left.\begin{array}{l}
\dot{e}_{1}(t)=-e_{1}(t) \\
\dot{e}_{2}(t)=-e_{2}(t) \\
\dot{e}_{3}(t)=-e_{3}(t) \\
\dot{e}_{4}(t)=-e_{4}(t) \\
\dot{e}_{5}(t)=-e_{5}(t) \\
\dot{e}_{6}(t)=-e_{6}(t)
\end{array}\right\}
$$

Thus, by Lyapunov stability theory, above error dynamical system is stable.

\section{Numerical Simulations for Synchronization}

For the parameters involved in the system under investigation $\mu=1, g_{1}=2$, $g_{2}=3$ with the initial conditions for master and slave systems

$$
\left[x_{1}(0), x_{2}(0), x_{3}(0), x_{4}(0), x_{5}(0), x_{6}(0)\right]=[2.5,0.8,-2.5,0.2,0.8,0.4]
$$

and $\left[y_{1}(0), y_{2}(0), y_{3}(0), y_{4}(0), y_{5}(0), y_{6}(0)\right]=[3.5,0.2,-0.8,0.4,0.3,2]$, respectively. We have simulated the system under consideration using mathematica. Phase portraits and time series analysis of master and slave system 
are the witness of irregular behavior of the system (see figures 1,2, 3, and $4)$. And for $\left[e_{1}(0), e_{2}(0), e_{3}(0), e_{4}(0), e_{5}(0), e_{6}(0)\right]=[1,-0.6,1.7,0.2,-0.5,1.6]$ convergence diagrams of errors are the witness of achieving synchronization between master and slave system (see figures 5, 6, 7, 8, 9, 10 and 11).

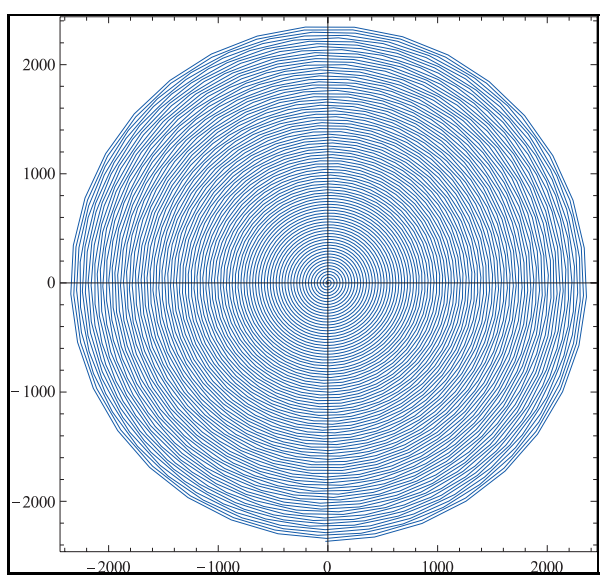

Fig. 1 Phase portrait of master system

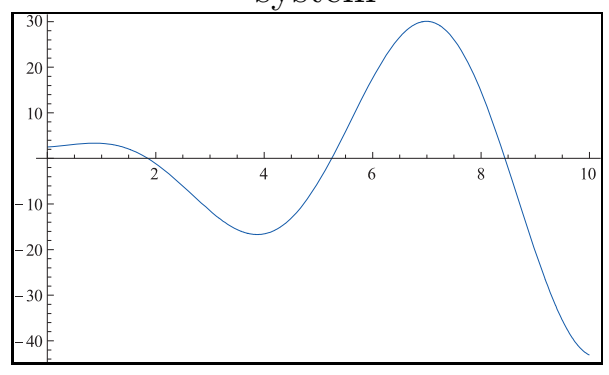

Fig. 3 Time series analysis of $x(t)$

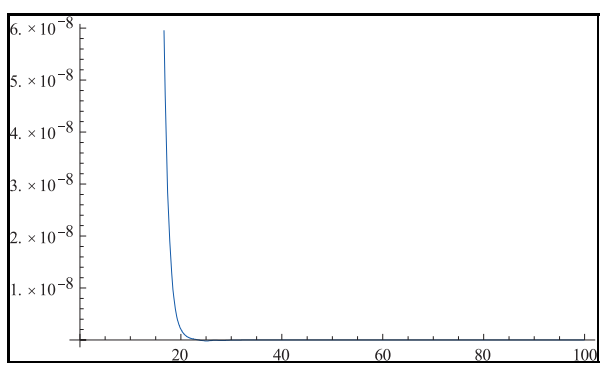

Fig. 5 Convergence of error $e_{1}(t)$

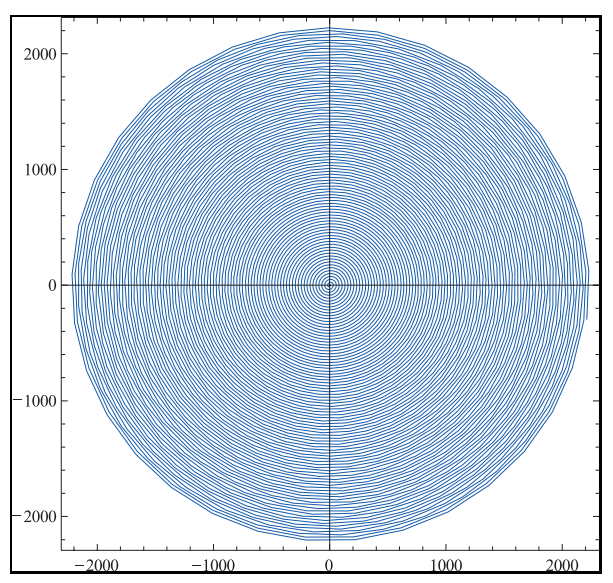

Fig. 2 Phase portrait of slave system

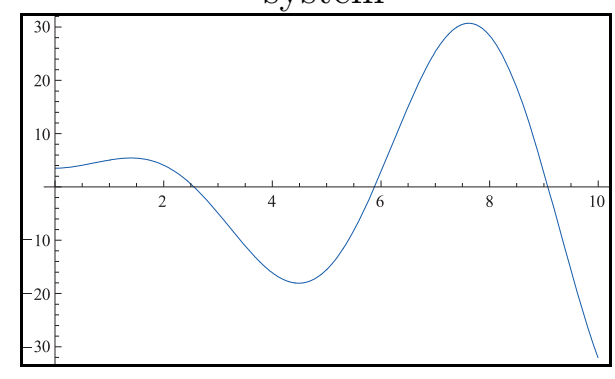

Fig. 4 Time series analysis of $y(t)$

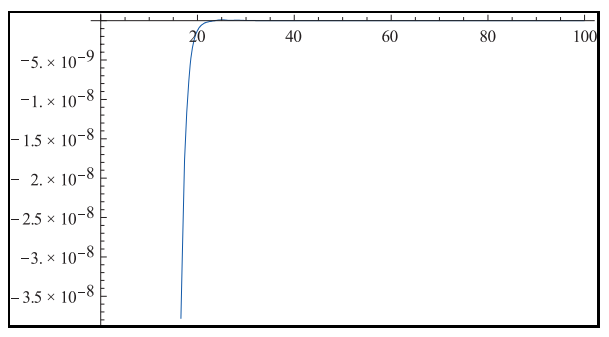

Fig. 6 Convergence of error $e_{2}(t)$ 


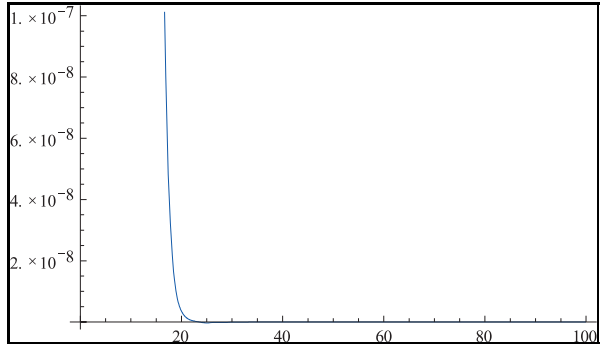

Fig. 7 Convergence of error $e_{3}(t)$

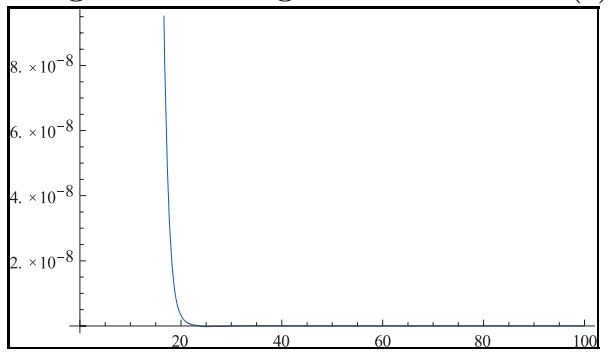

Fig. 9 Convergence of error $e_{5}(t)$

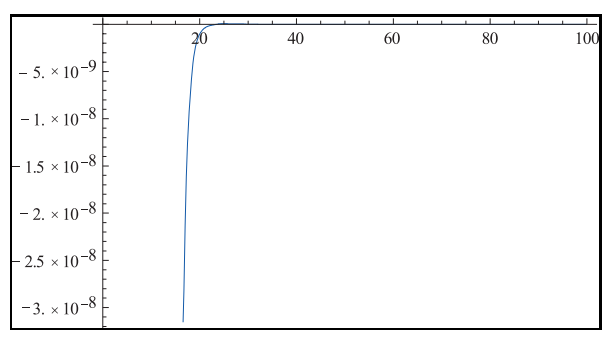

Fig. 8 Convergence of error $e_{4}(t)$

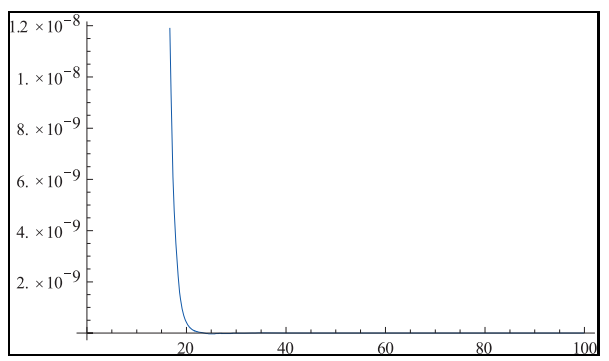

Fig. 10 Convergence of error $e_{6}(t)$

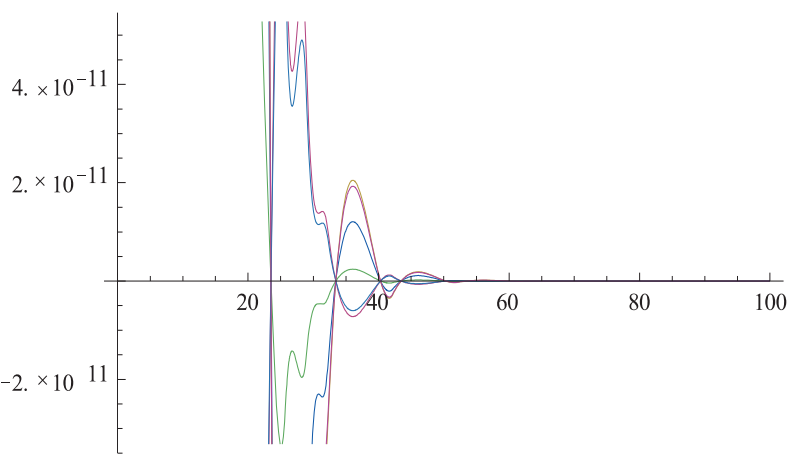

Fig. 11 Combined convergence of errors $e_{1}(t), e_{2}(t), e_{3}(t), e_{4}(t), e_{5}(t), e_{6}(t)$

\section{Conclusion}

An investigation on synchronization in restricted three body problem under the effect of radiation pressure via active control technique based on Lyapunov stability theory and Routh-Hurwitz criteria have been made. The results were validated by numerical simulations using Mathematica. 


\section{References}

[1] A. Pikovsky,M. Rosenblum, and J. Kurths. Synchronization: A Universal Concept Nonlinear Science. Cambridge University Press, 2002.

[2] L. Glass. Synchronization and rhythmic processes in physiology. Nature, 410:277,2001.

[3] S. Strogatz. Sync. Hyperion, 2003.

[4] L.M.Pecora and T.L. Carroll, Synchronization in chaotic systems, Phys. Rev. Lett. 64(1990): 821-824.

[5] Eulero L. Theoria Motus Lunae exhibens omnes eius inaequalitates. Academiae Imperialis Scientiarum: Petropolitanae, 1753. Russian translation: Euler L. New Lunar Movement Theory. Leningrad: Izd. Ak. Nauk USSR. 1934.

[6] Markeev AP. On three bodies problem and its exact solution. ISSEP. 1999.9. English translation: Markeev A.P. On three bodies problem and its exact solution. The Empire of Mathematics, 2000, 0(1).

[7] Poincare, Henri, New Methods of Celestial Mechanics, (AIP-1993).

[8] Sitnikov, K. [1960], Existence of oscillating motions in three-body problem, Sov. Phys. Dokl. 133, 303306.

[9] Arsen Dzhanoev and Alexander Loskutov, Stabilization of chaotic behaviour in the restricted three body problem, International Journal of Bifurcation and Chaos 17(10)(2007), 3603-3606.

[10] T.L. Liao and S.H. Lin, Adaptive control and synchronization of Lorenz systems, J. Franklin Inst. 336(1999): 92537.

[11] S.H. Chen and J. Lu, Synchronization of an uncertain unified system via adaptive control, Chaos Solitons \& Fractals 14(2002), 643-647.

[12] Z.M. Ge and Y.S. Chen, Adaptive synchronization of unidirectional and mutual coupled chaotic systems, Chaos Solitons \& Fractals 26(2005), 881888.

[13] C. Wang and S.S. Ge, Synchronization of two uncertain chaotic systems via adaptive backstepping, Int. J. Bifurcat. Chaos 11(2001), 17431751.

[14] C. Wang and S.S. Ge, Adaptive synchonizaion of uncertain chaotic systems via adaptive backstepping design, Chaos Solitons \& Fractals 12(2001), 1199-11206. 
[15] X. Tan, J. Zhang and Y. Yang, Synchronizing chaotic systems using backstepping design, Chaos Solitons \& Fractals 16(2003): 37-45.

[16] H.K. Chen, Synchronization of two different chaotic system: a new system and each of the dynamical systems Lorenz, Chen and Lu, Chaos Solitons \& Fractals 25(2005), 1049-1056.

[17] M.C. Ho and Y.C. Hung Synchronization two different systems by using generalized active control, Phys. Lett. A 301(2002), 424-428.

[18] M.T. Yassen, Chaos synchronization between two different chaotic systems using active control, Chaos Solitons \& Fractals 23(2005), 131-140.

[19] J.H. Park, Chaos synchronization between two different chaotic dynamical systems, Chaos Solitons \& Fractals 27(2006), 549-554.

[20] L. Huang, R. Feng and M. Wang, Snchronization of chaotic dynamical systems via nonlinear control, Phys. Lett. A 320(2004), 271-275.

[21] H.K. Chen, Global chaos synchronization of new chaoic systems via nonlinear control, Chaos Solitons \& Fractals 23(2005), 1245-1251.

[22] J.H. Park, Chaos synchronization of a chaotic system via nonlinear control, Chaos Solitons \& Fractals 25(2005), 549-584.

[23] Z.M. Ge, T.C. Yu and Y.S. Chen, Chaos synchronization of a horizontal platform system, J. Sound Vibrat 268(2003), 731-749.

[24] Gl. Wen and D. Xu, Observer-based control for full-state projective synchronization of a general class of chaotic maps in any dimension, Phys. Lett. A 330(2004), 420-425.

[25] Gl. Wen and D. Xu, Nonlinear observer control for full-state projective synchronization in chaotic continuous-time systems, Chaos Solitons \& Fractals 26(2005), 71-77.

[26] Z.M. Ge, J.W. Cheng and Y.S. Chen, Chaos anti-control and synchronization of three time scales brushless DC motor system, Chaos Solitons \& Fractals 22(2004), 1165-1182.

[27] Z.M. Ge and H.W. Wu, Chaos synchronization and chaos anti-control of a suspended track with moving load, J. Sound Vibrat 270(2004), 685-712.

[28] Z.M. Ge and C.C. Chen, Phase synchronization of coupled chaotic multiple time scale systems, Chaos Solitons \& Fractals 20(2004), 639-647. 
[29] Z.M. Ge and C.I., Control, anti-control and synchronization of chaos for an autonomous rotationa-machine system with time-delay, Chaos Solitons \& Fractals 23(2005), 1855-1864.

[30] Z.M. Ge, C.M. Chang and Y.S. Chen, Anti-control of chaos of single time scale brushless dc motors and chaos synchronization of different order systems, Chaos Solitons \& Fractals 27(2006), 1298-1315.

[31] A. Elipe and M. Lara, Periodic orbits in the Restricted three body Problem with Radiation Pressure, Celestial Mechanics and Dynamical Astronomy 68(1997), 1-11.

Received: September, 2012 